\title{
The Landscape of Histone Modifications in a High-Fat Diet-Induced Obese (DIO) Mouse Model
}

\author{
Minjia Tan, Litong Nie, Lin Shuai, Mingrui Zhu, Ping Liu \\ Shanghai Institute of Materia Medica, Chinese Academy of Sciences, China
}

Type 2 diabetes (T2D) is a major chronic healthcare concern worldwide. Emerging evidence suggests that a histonemodification-mediated epigenetic mechanism underlies T2D. Nevertheless, the dynamics of histone marks in T2D have not yet been carefully analyzed. Using a mass spectrometry-based label-free and chemical stable isotope labeling quantitative proteomic approach, we systematically profiled liver histone post-translational modifications (PTMs) in a prediabetic high-fat diet-induced obese (DIO) mouse model. We identified 170 histone marks, 30 of which were previously unknown. Interestingly, about 30\% of the histone marks identified in DIO mouse liver belonged to a set of recently reported lysine acylation modifications, including propionylation, butyrylation, malonylation, and succinylation, suggesting possible roles of these newly identified histone acylations in diabetes and obesity. These histone marks were detected without prior affinity enrichment with an antibody, demonstrating that the histone acylation marks are present at reasonably high stoichiometry. Fifteen histone marks differed in abundance in DIO mouse liver compared with liver from chow-fed mice in label-free quantification, and six histone marks in stable isotope labeling quantification. Analysis of hepatic histone modifications from metformintreated DIO mice revealed that metformin, a drug widely used for T2D, could reverse DIO-stimulated histone H3K36me2 in prediabetes, suggesting that this mark is likely associated with T2D development. Our study thus offers a comprehensive landscape of histone marks in a prediabetic mouse model, provides a resource for studying epigenetic functions of histone modifications in obesity and $\mathrm{T} 2 \mathrm{D}$, and suggest a new epigenetic mechanism for the physiological function of metformin. 